Acta Crystallographica Section C

Crystal Structure

Communications

ISSN 0108-2701

\section{2-Amino-2-thiazoline and its 1:1 organic salt with 2-naphthoxyacetic acid}

\section{Daniel E. Lynch}

School of Science and the Environment, Coventry University, Coventry CV1 5FB, England

Correspondence e-mail: apx106@coventry.ac.uk

Received 15 June 2004

Accepted 25 June 2004

Online 21 August 2004

The crystal structures of 2-amino-2-thiazoline, $\mathrm{C}_{3} \mathrm{H}_{6} \mathrm{~N}_{2} \mathrm{~S}$, and 2-amino-2-thiazolinium 2-naphthoxyacetate, $\mathrm{C}_{3} \mathrm{H}_{7} \mathrm{~N}_{2} \mathrm{~S}^{+}$.$\mathrm{C}_{12} \mathrm{H}_{9} \mathrm{O}_{3}{ }^{-}$, are reported. The structure of 2-amino-2-thiazoline consists of two unique molecules that construct a convoluted hydrogen-bonded ribbon involving $R_{2}^{2}(8)$ graph-set association via both $\mathrm{N}-\mathrm{H} \cdots \mathrm{N}$ and $\mathrm{N}-\mathrm{H} \cdots \mathrm{S}$ interactions. The organic salt structure consists of the two molecules associated via an $R_{2}^{2}(8)$ graph-set dimer through $\mathrm{N}-\mathrm{H} \cdots \mathrm{O}$ interactions, with the hydrogen-bonding network propagated via additional $\mathrm{N}-\mathrm{H}$. . O three-centre interactions from the second 2-amine $\mathrm{H}$ atom.

\section{Comment}

2-Amino-2-thiazoline has been reported as a potential inducer of the reverse transformation of tumour cells, with the mechanism for anticancer action depending on strong metalligand binding via the N atoms (Brugarolas \& Gosálvez, 1982). Alternatively, the placement of the $\mathrm{N}$ atoms in this molecule also makes it suitable for association with carboxylic acids, and four subsequent crystal structures have been reported (Lynch et al., 1998; Lynch, Cooper et al., 1999; Lynch, Nicholls et al., 1999). Such structures are part of a broader study of complexes of carboxylic acids with 2-aminothiazole derivatives that has thus far resulted in the characterization of 19 published crystal structures, with three others published recently (Lynch et al., 2004). Although the structure of 2-aminothiazole was published by Caranoni \& Reboul (1982), the structure of 2-amino-2-thiazoline has not been reported; the structure of this compound, (I), is reported here. 2-Naphthoxyacetic acid is used as a plant hormone to promote growth of roots on clippings and to prevent fruit from falling prematurely, although stunted growth results if it is used in excess (The Merck Index, 2001). 2-Naphthoxyacetic acid is related in structure to phenoxyacetic acid, whose chloro derivatives have been used extensively by the author for complexing with carboxylic acids (Lynch, Cooper et al., 1999) and should thus have comparable structural properties.
Furthermore, the Cambridge Structural Database (Allen, 2002) contains only four previously reported crystal structures containing the compound, of which two are the parent structure (Howie et al., 2001), thus more structures containing 2-naphthoxyacetic acid are required. For these reasons, the structure of the 1:1 organic salt of (I) with 2-naphthoxyacetic acid is also reported here, viz. (II).

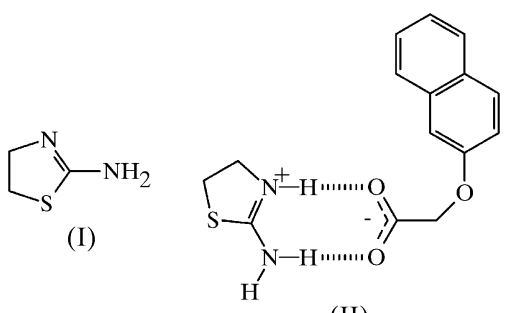

(II)

Compound (I) packs with two unique thiazoline molecules associated in a hydrogen-bonded $R_{2}^{2}(8)$ graph-set dimer (Etter, 1990) via $\mathrm{N}-\mathrm{H} \cdots \mathrm{N}$ interactions (Fig. 1). The hydrogen-bonding network is then extended by $\mathrm{N}-\mathrm{H} \cdots \mathrm{S}$ interactions, resulting in further $R_{2}^{2}(8)$ graph-set arrangements. Hydrogen-bonding associations for this compound are listed in Table 1. Together, these interactions create a convoluted

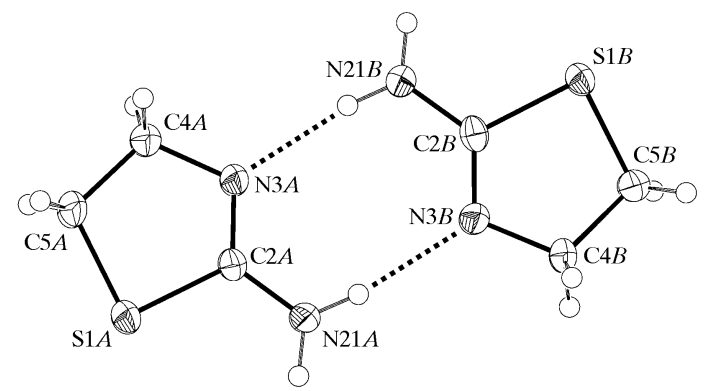

Figure 1

A view of the asymmetric unit and atom-numbering scheme of (I). Displacement ellipsoids are drawn at the $50 \%$ probability level. Broken lines indicate intramolecular hydrogen bonds.

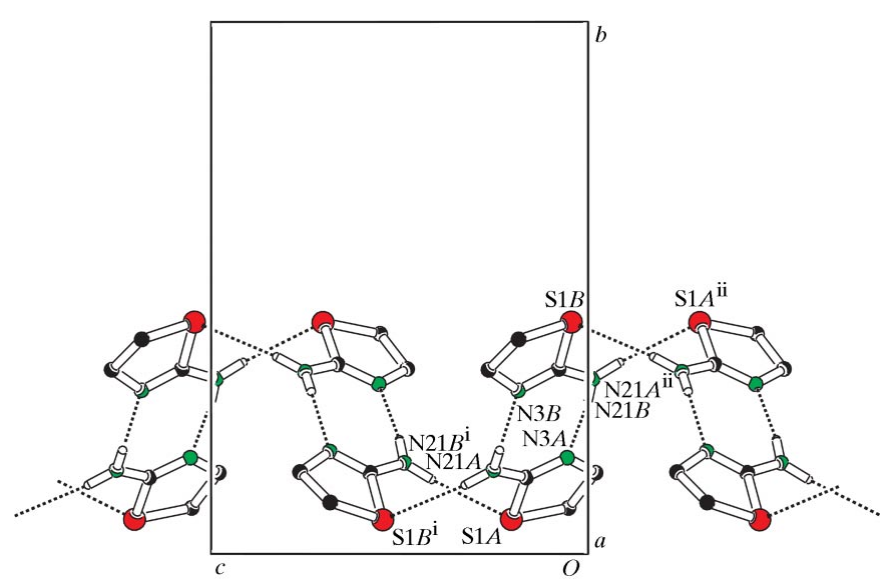

Figure 2

A packing diagram for (I). [Symmetry codes: (i) $x-\frac{1}{2},-y+\frac{1}{2}, z+\frac{1}{2}$; (ii) $x+\frac{1}{2},-y+\frac{1}{2}, z-\frac{1}{2}$.] 
hydrogen-bonded ribbon that runs in the direction of the $a c$ axis diagonal (Fig. 2). The incorporation of the $\mathrm{S}$ atoms into the hydrogen-bonding network is not observed in the structure of 2-aminothiazole but is seen in the structure of a related 2-aminothiazole derivative, viz. 2-amino-4-(4-bisphenyl)-1,3thiazole (Lynch et al., 2002). In (I), there is a single $\mathrm{S} \cdots \mathrm{S}$ close contact $[3.520(5) \AA]$ between atom S1B and the symmetryequivalent atom at $(2-x, 1-y,-z)$.

The structure of (II) comprises the organic salt of a nonplanar acetate molecule and a protonated thiazoline molecule arranged in a packing mode commonly observed for these types of molecules. In contrast to its planar parent structure, the acetate chain of the naphthoxyacetate molecule in (II) adopts an anticlinical (or hooked) arrangement, as classified for phenoxyacetic acids (Smith \& Kennard, 1979) and defined by the $\mathrm{C} 2 B-\mathrm{O} 11 B-\mathrm{C} 12 B-\mathrm{C} 13 B$ torsion angle [92.8 (2) Fig. 3]. Packing with the thiazoline molecule has an associated effect on (2,4,5-trichlorophenoxy)acetic acid, whose structure is planar in the parent compound but hooked in the salt complex (Lynch, Cooper et al., 1999). The components of (II), like those of the vast majority of adducts/organic salts comprising a 2-amino-heterocycle and a carboxylic acid molecule, associate via an unsymmetrical $R_{2}^{2}(8)$ graph-set dimer between the $\mathrm{N}=\mathrm{C}-\mathrm{NH}_{2}$ site and the carboxylate group (Fig. 4). In general, this association is unsymmetrical in that the $\mathrm{N} 3 A \cdots \mathrm{O} 14 B$ distance, or equivalent, is (apart from a very few cases) shorter than the $\mathrm{N} 21 A \cdots \mathrm{O} 15 B$ distance, although the values listed in Table 2 indicate that the structure of (I) is one of the very few exceptions where the opposite has occurred. Another common feature of this association is the inconsistency of the $\mathrm{C} 2 A-\mathrm{N} 21 A[1.302(2) \AA]$ and $\mathrm{C} 2 A-$ $\mathrm{N} 3 A[1.324$ (2) $\AA$ ] bond lengths, as previously highlighted (Lynch et al., 2000). The propagation of the hydrogen-bonding network via the $\mathrm{N} 21 A-\mathrm{H} 22 A \cdots \mathrm{O} 14 B(x, y-1, z)$ interaction has also been observed previously for these types of systems

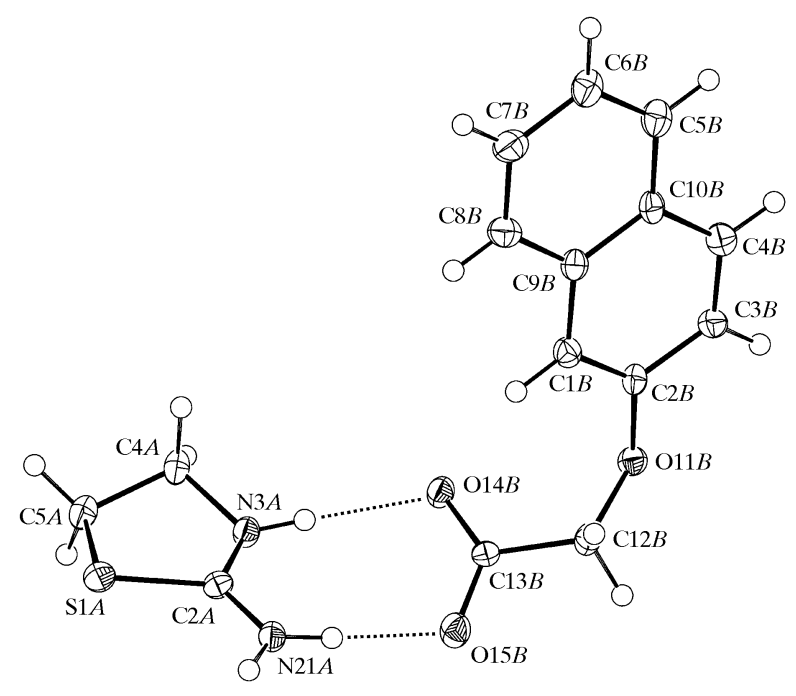

Figure 3

A view of the asymmetric unit and atom-numbering scheme of (II). Displacement ellipsoids are drawn at the $50 \%$ probability level. Broken lines indicate intramolecular hydrogen bonds.

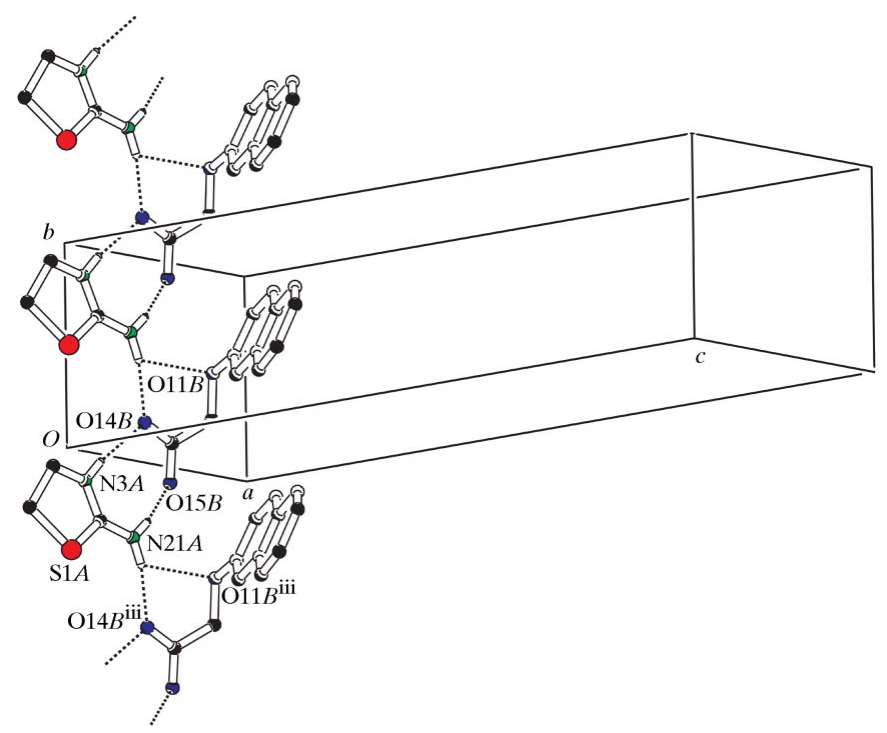

Figure 4

A packing diagram for (II). [Symmetry code: (iii) $x, y-1, z$.]

(Lynch, Nicholls et al., 1999), although the additional interaction with atom $\mathrm{O} 11 B$ is not common amongst complexes of 2-aminothiazole derivatives and phenoxyacetic acids (Lynch, Cooper et al., 1999).

The structure of (II) is actually the eighth known complex of a carboxylic acid with (I), with three others currently unpublished (Lynch et al., 2004). Elucidation of the structure of (I) is important because, as highlighted above, when collecting data on the inconsistencies in the bond distances across the $\mathrm{N}=\mathrm{C}-\mathrm{NH}_{2}$ site for any type of complexed 2amino-heterocyclic compound, it is important to compare bond distances against those of the parent structure. For example, compare the $\mathrm{C} 2 A-\mathrm{N} 21 A$ and $\mathrm{C} 2 A-\mathrm{N} 3 A$ distances listed above with those for (I), viz. $1.348(5) / 1.267$ (5) and 1.351 (5)/1.276 (5) $\AA$ for molecules $A$ and $B$, respectively. The mean respective distances for the seven complex structures are 1.305 (5) and 1.314 (5) $\AA$. Also of interest is the N3-C2N21 (or equivalent) angle, which decreases upon association with a carboxylic acid. Compare, for (I), values of 124.8 (3) and $125.8(3)^{\circ}$ with that of $124.53(17)^{\circ}$ in (II) [the mean angle over the eight structures is $\left.124.0(5)^{\circ}\right]$. In one or two instances where $\mathrm{N} 1 A$ is a quaternary $\mathrm{N}$ atom, it might be suitable to suggest that the $\mathrm{N} 1 A-\mathrm{C} 2 A$ double bond has moved to $\mathrm{C} 2 A-$ $\mathrm{N} 21 A$, but this simple 'pushing of the double bond around' does not fit a significant portion of the available data. It is the intention of the author to publish such findings in a dedicated paper, but not without each of the parent structures and a supportive list of different complexes, which the structures in this paper add to.

\section{Experimental}

Crystals of (I) were grown from an ethanol solution. For (II), equimolar amounts of (I) and 2-naphthoxyacetic acid were refluxed in ethanol for $20 \mathrm{~min}$. Crystals of (II) were grown by slow evaporation of the reaction solution. 


\section{Compound (I) \\ Crystal data}

$\mathrm{C}_{3} \mathrm{H}_{6} \mathrm{~N}_{2} \mathrm{~S}$
$M_{r}=102.17$
Monoclinic, $P 2_{1} / n$
$a=5.8980(5) \AA$
$b=14.8324(12) \AA$
$c=10.7092(8) \AA$
$\beta=101.974(4)^{\circ}$
$V=916.47(13) \AA^{3}$
$Z=8$

Data collection

Nonius KappaCCD area-detector diffractometer

$\varphi$ and $\omega$ scans

Absorption correction: multi-scan

(SORTAV; Blessing, 1995)

$T_{\min }=0.826, T_{\max }=0.948$

10396 measured reflections

\section{Refinement}

Refinement on $F^{2}$

$R\left[F^{2}>2 \sigma\left(F^{2}\right)\right]=0.061$

$w R\left(F^{2}\right)=0.160$

$S=1.02$

2101 reflections

109 parameters

Table 1

Hydrogen-bonding geometry $\left(\AA{ }^{\circ}{ }^{\circ}\right)$ for $(\mathrm{I})$.

\begin{tabular}{lllll}
\hline$D-\mathrm{H} \cdots A$ & $D-\mathrm{H}$ & $\mathrm{H} \cdots A$ & $D \cdots A$ & $D-\mathrm{H} \cdots A$ \\
\hline $\mathrm{N} 21 A-\mathrm{H} 21 A \cdots \mathrm{N} 3 B$ & 0.88 & 2.09 & $2.950(5)$ & 164 \\
$\mathrm{~N} 21 A-\mathrm{H} 22 A \cdots \mathrm{S} 1 B^{\mathrm{i}}$ & 0.88 & 2.75 & $3.575(3)$ & 156 \\
$\mathrm{~N} 21 B-\mathrm{H} 21 B \cdots \mathrm{N} 3 A$ & 0.88 & 2.04 & $2.916(5)$ & 171 \\
$\mathrm{~N} 21 B-\mathrm{H} 22 B \cdots \mathrm{S} 1 A^{\text {ii }}$ & 0.88 & 2.70 & $3.526(3)$ & 156
\end{tabular}

Symmetry codes: (i) $x-\frac{1}{2},-y+\frac{1}{2}, z+\frac{1}{2}$; (ii) $x+\frac{1}{2},-y+\frac{1}{2}, z-\frac{1}{2}$.

\section{Compound (II)}

\section{Crystal data}

$$
\begin{aligned}
& \mathrm{C}_{3} \mathrm{H}_{7} \mathrm{~N}_{2} \mathrm{~S}^{+} \cdot \mathrm{C}_{12} \mathrm{H}_{9} \mathrm{O}_{3}{ }^{-} \\
& M_{r}=304.36 \\
& \text { Monoclinic, } P 2_{1} / c \\
& a=8.3669(2) \AA \\
& b=6.3707(1) \AA \\
& c=26.3457(6) \AA \\
& \beta=92.1992(9)^{\circ} \\
& V=1403.27(5) \AA^{3} \\
& Z=4
\end{aligned}
$$

\section{Data collection}

Nonius KappaCCD area-detector diffractometer

$\varphi$ and $\omega$ scans

Absorption correction: multi-scan (SORTAV; Blessing, 1995)

$T_{\min }=0.710, T_{\max }=0.990$

15534 measured reflections

\section{Refinement}

Refinement on $F^{2}$

$R\left[F^{2}>2 \sigma\left(F^{2}\right)\right]=0.046$

$w R\left(F^{2}\right)=0.121$

$S=1.02$

3183 reflections

194 parameters

$\mathrm{H}$ atoms treated by a mixture of independent and constrained refinement

\author{
$D_{x}=1.481 \mathrm{Mg} \mathrm{m}^{-3}$ \\ Mo $K \alpha$ radiation \\ Cell parameters from 8580 \\ reflections \\ $\theta=2.9-27.5^{\circ}$ \\ $\mu=0.53 \mathrm{~mm}^{-1}$ \\ $T=120(2) \mathrm{K}$ \\ Prism, yellow \\ $0.20 \times 0.20 \times 0.10 \mathrm{~mm}$
}

\author{
2101 independent reflections \\ 1149 reflections with $I>2 \sigma(I)$ \\ $R_{\text {int }}=0.112$ \\ $\theta_{\max }=27.5^{\circ}$ \\ $h=-7 \rightarrow 7$ \\ $k=-19 \rightarrow 19$ \\ $l=-13 \rightarrow 13$
}

$\mathrm{H}$-atom parameters constrained $w=1 /\left[\sigma^{2}\left(F_{o}^{2}\right)+(0.0802 P)^{2}\right]$

where $P=\left(F_{o}^{2}+2 F_{c}^{22}\right) / 3$

$(\Delta / \sigma)_{\max }<0.001$

$\Delta \rho_{\max }=0.49{\mathrm{e} \AA^{-3}}^{-3}$

$\Delta \rho_{\min }=-0.41{\mathrm{e} \AA^{-3}}^{-3}$
Table 2

Hydrogen-bonding geometry $\left(\AA,^{\circ}\right)$ for (II).

\begin{tabular}{lllll}
\hline$D-\mathrm{H} \cdots A$ & $D-\mathrm{H}$ & $\mathrm{H} \cdots A$ & $D \cdots A$ & $D-\mathrm{H} \cdots A$ \\
\hline $\mathrm{N} 21 A-\mathrm{H} 21 A \cdots \mathrm{O} 15 B$ & 0.88 & 1.88 & $2.754(2)$ & 171 \\
$\mathrm{~N} 21 A-\mathrm{H} 22 A \cdots \mathrm{O} 14 B^{\text {iii }}$ & 0.88 & 1.93 & $2.787(2)$ & 166 \\
$\mathrm{~N} 21 A-\mathrm{H} 22 A \cdots \mathrm{O} 11 B^{\text {iii }}$ & 0.88 & 2.56 & $2.965(2)$ & 109 \\
$\mathrm{~N} 3 A-\mathrm{H} 3 A \cdots \mathrm{O} 14 B$ & $0.87(2)$ & $2.01(2)$ & $2.871(2)$ & $170(2)$
\end{tabular}

Symmetry code: (iii) $x, y-1, z$.

All $\mathrm{H}$ atoms, except for the $\mathrm{H}$ atom on the $\mathrm{N}^{+}$ion in (II), were included in the refinement at calculated positions in the riding-model approximation, with $\mathrm{N}-\mathrm{H}$ distances of $0.88 \AA$, and $\mathrm{C}-\mathrm{H}$ distances of 0.95 (aromatic $\mathrm{H}$ atoms) and $0.99 \AA\left(\mathrm{CH}_{2} \mathrm{H}\right.$ atoms). The $U_{\text {iso }}(\mathrm{H})$ values were set at $1.25 U_{\text {eq }}$ of the carrier atom. The $\mathrm{H}$ atom on the $\mathrm{N}^{+}$ ion was located in a difference synthesis and both the positional and displacement parameters were refined. A high $R_{\text {int }}$ value for (I) was the result of weak high-angle data.

For both compounds, data collection: DENZO (Otwinowski \& Minor, 1997) and COLLECT (Hooft, 1998); cell refinement: DENZO and COLLECT; data reduction: DENZO, SCALEPACK (Otwinowski \& Minor, 1997) and COLLECT; program(s) used to solve structure: SHELXS97 (Sheldrick, 1997); program(s) used to refine structure: SHELXL97 (Sheldrick, 1997); molecular graphics: PLUTON94 (Spek, 1994) and PLATON97 (Spek, 1997); software used to prepare material for publication: SHELXL97 (Sheldrick, 1997).

The authors thank the EPSRC National Crystallography Service (Southampton, England).

Supplementary data for this paper are available from the IUCr electronic archives (Reference: SK1737). Services for accessing these data are described at the back of the journal.

\section{References}

$D_{x}=1.441 \mathrm{Mg} \mathrm{m}^{-3}$

Mo $K \alpha$ radiation

Cell parameters from 4067

reflections

$\theta=2.9-27.5^{\circ}$

$\mu=0.24 \mathrm{~mm}^{-1}$

$T=120(2) \mathrm{K}$

Plate, colourless

$0.32 \times 0.10 \times 0.04 \mathrm{~mm}$

3183 independent reflections 2546 reflections with $I>2 \sigma(I)$

$R_{\text {int }}=0.091$

$\theta_{\max }=27.4^{\circ}$

$h=-10 \rightarrow 10$

$k=-7 \rightarrow 8$

$l=-34 \rightarrow 34$

$$
\begin{gathered}
w=1 /\left[\sigma^{2}\left(F_{o}^{2}\right)+(0.0503 P)^{2}\right. \\
+0.8055 P] \\
\text { where } P=\left(F_{o}^{2}+2 F_{c}^{2}\right) / 3 \\
(\Delta / \sigma)_{\max }<0.001 \\
\Delta \rho_{\max }=0.25 \mathrm{e}^{-3} \\
\Delta \rho_{\min }=-0.34 \AA^{-3}
\end{gathered}
$$

Allen, F. H. (2002). Acta Cryst. B58, 380-388.

Blessing, R. H. (1995). Acta Cryst. A51, 33-37.

Brugarolas, A. \& Gosálvez, M. (1982). Recent Results Cancer Res. 80, 346-350. Caranoni, P. C. \& Reboul, J. P. (1982). Acta Cryst. B38, 1255-1259.

Etter, M. C. (1990). Acc. Chem. Res. 23, 120-126.

Hooft, R. (1998). COLLECT. Nonius BV, Delft, The Netherlands.

Howie, R. A., Skakle, J. M. S. \& Wardell, S. M. S. V. (2001). Acta Cryst. E57, o72-o74.

Lynch, D. E., Barfield, J., Frost, J., Antrobus, R. \& Simmons, J. (2004). Cryst. Eng. 6, 109-122.

Lynch, D. E., Cooper, C. J., Chauhan, V., Smith, G., Healy, P. \& Parsons, S. (1999). Aust. J. Chem. 52, 695-703.

Lynch, D. E., Daly, D. \& Parsons, S. (2000). Acta Cryst. C56, 1478-1479.

Lynch, D. E., McClenaghan, I., Light, M. E. \& Coles, S. J. (2002). Cryst. Eng. 5, 123-136.

Lynch, D. E., Nicholls, L. J., Smith, G., Byriel, K. A. \& Kennard, C. H. L. (1999). Acta Cryst. B55, 758-766.

Lynch, D. E., Smith, G., Byriel, K. A. \& Kennard, C. H. L. (1998). Aust. J. Chem. 51, 587-592.

Otwinowski, Z. \& Minor, W. (1997). Methods in Enzymology, Vol. 276, Macromolecular Crystallography, Part A, edited by C. W. Carter Jr \& R. M. Sweet, pp. 307-326. New York: Academic Press.

Sheldrick, G. M. (1997). SHELXS97 and SHELXL97. University of Göttingen, Germany.

Smith, G. \& Kennard, C. H. L. (1979). J. Agric. Food Chem. 27, 779-787.

Spek, A. L. (1994). PLUTON94. University of Utrecht, The Netherlands.

Spek, A. L. (1997). PLATON97. University of Utrecht, The Netherlands.

The Merck Index (2001). 13th ed. Whitehouse Station: Merck and Co. Inc. 


\section{supporting information}

Acta Cryst. (2004). C60, o677-o679 [doi:10.1107/S0108270104015604]

\section{2-Amino-2-thiazoline and its 1:1 organic salt with 2-naphthoxyacetic acid}

\section{Daniel E. Lynch}

\section{Computing details}

For both compounds, data collection: DENZO (Otwinowski \& Minor, 1997) and COLLECT (Hooft, 1998); cell refinement: DENZO and COLLECT; data reduction: DENZO, SCALEPACK (Otwinowski \& Minor, 1997) and COLLECT; program(s) used to solve structure: SHELXS97 (Sheldrick, 1997); program(s) used to refine structure: SHELXL97 (Sheldrick, 1997); molecular graphics: PLUTON94 (Spek, 1994) and PLATON97 (Spek, 1997); software used to prepare material for publication: SHELXL97 (Sheldrick, 1997).

\section{(I) 2-Amino-2-thiazoline}

Crystal data

\section{$\mathrm{C}_{3} \mathrm{H}_{6} \mathrm{~N}_{2} \mathrm{~S}$}

$M_{r}=102.17$

Monoclinic, $P 2_{1} / n$

Hall symbol: -P $2 \mathrm{yn}$

$a=5.8980(5) \AA$

$b=14.8324(12) \AA$

$c=10.7092(8) \AA$

$\beta=101.974(4)^{\circ}$

$V=916.47(13) \AA^{3}$

$Z=8$

\section{Data collection}

Nonius KappaCCD area-detector diffractometer

Radiation source: Nonius FR591 rotating anode Graphite monochromator

Detector resolution: 9.091 pixels $\mathrm{mm}^{-1}$

$\varphi$ and $\omega$ scans

Absorption correction: multi-scan

(SORTAV; Blessing, 1995)

$T_{\min }=0.826, T_{\max }=0.948$

Refinement

Refinement on $F^{2}$

Least-squares matrix: full

$R\left[F^{2}>2 \sigma\left(F^{2}\right)\right]=0.061$

$w R\left(F^{2}\right)=0.160$

$S=1.02$

2101 reflections

109 parameters

0 restraints
$F(000)=432$

$D_{\mathrm{x}}=1.481 \mathrm{Mg} \mathrm{m}^{-3}$

Mo $K \alpha$ radiation, $\lambda=0.71073 \AA$

Cell parameters from 8580 reflections

$\theta=2.9-27.5^{\circ}$

$\mu=0.53 \mathrm{~mm}^{-1}$

$T=120 \mathrm{~K}$

Prism, yellow

$0.20 \times 0.20 \times 0.10 \mathrm{~mm}$

10396 measured reflections

2101 independent reflections

1149 reflections with $I>2 \sigma(I)$

$R_{\text {int }}=0.112$

$\theta_{\max }=27.5^{\circ}, \theta_{\min }=3.4^{\circ}$

$h=-7 \rightarrow 7$

$k=-19 \rightarrow 19$

$l=-13 \rightarrow 13$

Primary atom site location: structure-invariant direct methods

Secondary atom site location: difference Fourier map

Hydrogen site location: inferred from neighbouring sites

$\mathrm{H}$-atom parameters constrained 
$w=1 /\left[\sigma^{2}\left(F_{\mathrm{o}}^{2}\right)+(0.0802 P)^{2}\right]$

where $P=\left(F_{\mathrm{o}}^{2}+2 F_{\mathrm{c}}^{2}\right) / 3$

$(\Delta / \sigma)_{\max }<0.001$
$\Delta \rho_{\max }=0.49 \mathrm{e} \AA^{-3}$

$\Delta \rho_{\min }=-0.41 \mathrm{e} \AA^{-3}$

Fractional atomic coordinates and isotropic or equivalent isotropic displacement parameters $\left(\AA^{2}\right)$

\begin{tabular}{lllll}
\hline & $x$ & $y$ & $z$ & $U_{\text {iso }} / U_{\text {eq }}$ \\
\hline S1A & $-0.02730(17)$ & $0.06496(7)$ & $0.20321(9)$ & $0.0269(3)$ \\
C2A & $0.1754(6)$ & $0.1438(2)$ & $0.1644(4)$ & $0.0219(8)$ \\
N21A & $0.3725(6)$ & $0.1576(2)$ & $0.2517(3)$ & $0.0295(8)$ \\
H21A & 0.4788 & 0.1946 & 0.2348 & $0.037^{*}$ \\
H22A & 0.3949 & 0.1296 & 0.3257 & $0.037^{*}$ \\
N3A & $0.1270(5)$ & $0.1808(2)$ & $0.0554(3)$ & $0.0263(8)$ \\
C4A & $-0.0968(7)$ & $0.1525(3)$ & $-0.0198(4)$ & $0.0271(9)$ \\
H41A & -0.1959 & 0.2062 & -0.0434 & $0.034^{*}$ \\
H42A & -0.0732 & 0.1238 & -0.0996 & $0.034^{*}$ \\
C5A & $-0.2206(7)$ & $0.0864(3)$ & $0.0520(4)$ & $0.0309(10)$ \\
H51A & -0.3679 & 0.1127 & 0.0655 & $0.039^{*}$ \\
H52A & -0.2554 & 0.0297 & 0.0031 & $0.039^{*}$ \\
S1B & $0.77862(17)$ & $0.43581(7)$ & $0.04456(9)$ & $0.0246(3)$ \\
C2B & $0.5938(6)$ & $0.3476(2)$ & $0.0781(4)$ & $0.0228(8)$ \\
N21B & $0.4037(5)$ & $0.3289(2)$ & $-0.0124(3)$ & $0.0289(8)$ \\
H21B & 0.3088 & 0.2856 & 0.0002 & $0.036^{*}$ \\
H22B & 0.3747 & 0.3599 & -0.0840 & $0.036^{*}$ \\
N3B & $0.6540(5)$ & $0.3076(2)$ & $0.1853(3)$ & $0.0260(8)$ \\
C4B & $0.8603(6)$ & $0.3461(3)$ & $0.2639(4)$ & $0.0270(9)$ \\
H41B & 0.9621 & 0.2972 & 0.3062 & $0.034^{*}$ \\
H42B & 0.8163 & 0.3841 & 0.3311 & $0.034^{*}$ \\
C5B & $0.9918(7)$ & $0.4032(3)$ & $0.1840(4)$ & $0.0306(10)$ \\
H51B & 1.1178 & 0.3678 & 0.1592 & $0.038^{*}$ \\
H52B & 1.0599 & 0.4571 & 0.2322 & $0.038^{*}$ \\
& & & & \\
\hline
\end{tabular}

Atomic displacement parameters $\left(\AA^{2}\right)$

\begin{tabular}{lllllll}
\hline & $U^{11}$ & $U^{22}$ & $U^{33}$ & $U^{12}$ & $U^{13}$ & $U^{23}$ \\
\hline S1A & $0.0240(6)$ & $0.0311(6)$ & $0.0266(6)$ & $-0.0060(4)$ & $0.0077(4)$ & $0.0016(4)$ \\
C2A & $0.020(2)$ & $0.0226(19)$ & $0.024(2)$ & $-0.0013(15)$ & $0.0066(17)$ & $-0.0004(16)$ \\
N21A & $0.0234(18)$ & $0.0421(19)$ & $0.0225(18)$ & $-0.0074(16)$ & $0.0039(14)$ & $0.0078(15)$ \\
N3A & $0.0228(18)$ & $0.0346(18)$ & $0.0217(18)$ & $-0.0079(14)$ & $0.0049(14)$ & $0.0011(15)$ \\
C4A & $0.024(2)$ & $0.031(2)$ & $0.026(2)$ & $-0.0034(17)$ & $0.0041(17)$ & $0.0014(18)$ \\
C5A & $0.020(2)$ & $0.043(3)$ & $0.030(2)$ & $-0.0073(18)$ & $0.0067(19)$ & $0.0003(19)$ \\
S1B & $0.0229(6)$ & $0.0269(5)$ & $0.0253(6)$ & $0.0042(4)$ & $0.0079(4)$ & $-0.0014(4)$ \\
C2B & $0.019(2)$ & $0.0241(19)$ & $0.028(2)$ & $0.0016(16)$ & $0.0109(17)$ & $0.0035(17)$ \\
N21B & $0.0272(19)$ & $0.0346(18)$ & $0.0232(18)$ & $0.0097(14)$ & $0.0014(15)$ & $-0.0031(15)$ \\
N3B & $0.0213(18)$ & $0.0331(18)$ & $0.0229(18)$ & $0.0037(14)$ & $0.0034(15)$ & $-0.0018(14)$ \\
C4B & $0.020(2)$ & $0.032(2)$ & $0.028(2)$ & $0.0064(17)$ & $0.0043(17)$ & $-0.0013(18)$ \\
C5B & $0.024(2)$ & $0.040(2)$ & $0.027(2)$ & $0.0027(18)$ & $0.0037(18)$ & $-0.0074(19)$ \\
& & & & & &
\end{tabular}


Geometric parameters $\left(\AA,{ }^{\circ}\right)$

\begin{tabular}{|c|c|c|c|}
\hline $\mathrm{S} 1 \mathrm{~A}-\mathrm{C} 2 \mathrm{~A}$ & $1.782(4)$ & $\mathrm{S} 1 \mathrm{~B}-\mathrm{C} 2 \mathrm{~B}$ & $1.786(4)$ \\
\hline $\mathrm{S} 1 \mathrm{~A}-\mathrm{C} 5 \mathrm{~A}$ & $1.805(4)$ & $\mathrm{S} 1 \mathrm{~B}-\mathrm{C} 5 \mathrm{~B}$ & $1.807(4)$ \\
\hline $\mathrm{C} 2 \mathrm{~A}-\mathrm{N} 3 \mathrm{~A}$ & $1.267(5)$ & $\mathrm{C} 2 \mathrm{~B}-\mathrm{N} 3 \mathrm{~B}$ & $1.276(5)$ \\
\hline $\mathrm{C} 2 \mathrm{~A}-\mathrm{N} 21 \mathrm{~A}$ & $1.348(5)$ & $\mathrm{C} 2 \mathrm{~B}-\mathrm{N} 21 \mathrm{~B}$ & $1.351(5)$ \\
\hline $\mathrm{N} 21 \mathrm{~A}-\mathrm{H} 21 \mathrm{~A}$ & 0.88 & $\mathrm{~N} 21 \mathrm{~B}-\mathrm{H} 21 \mathrm{~B}$ & 0.88 \\
\hline $\mathrm{N} 21 \mathrm{~A}-\mathrm{H} 22 \mathrm{~A}$ & 0.88 & $\mathrm{~N} 21 \mathrm{~B}-\mathrm{H} 22 \mathrm{~B}$ & 0.88 \\
\hline $\mathrm{N} 3 \mathrm{~A}-\mathrm{C} 4 \mathrm{~A}$ & $1.458(5)$ & $\mathrm{N} 3 \mathrm{~B}-\mathrm{C} 4 \mathrm{~B}$ & $1.446(5)$ \\
\hline $\mathrm{C} 4 \mathrm{~A}-\mathrm{C} 5 \mathrm{~A}$ & $1.522(5)$ & $\mathrm{C} 4 \mathrm{~B}-\mathrm{C} 5 \mathrm{~B}$ & $1.525(5)$ \\
\hline $\mathrm{C} 4 \mathrm{~A}-\mathrm{H} 41 \mathrm{~A}$ & 0.99 & $\mathrm{C} 4 \mathrm{~B}-\mathrm{H} 41 \mathrm{~B}$ & 0.99 \\
\hline $\mathrm{C} 4 \mathrm{~A}-\mathrm{H} 42 \mathrm{~A}$ & 0.99 & $\mathrm{C} 4 \mathrm{~B}-\mathrm{H} 42 \mathrm{~B}$ & 0.99 \\
\hline $\mathrm{C} 5 \mathrm{~A}-\mathrm{H} 51 \mathrm{~A}$ & 0.99 & $\mathrm{C} 5 \mathrm{~B}-\mathrm{H} 51 \mathrm{~B}$ & 0.99 \\
\hline $\mathrm{C} 5 \mathrm{~A}-\mathrm{H} 52 \mathrm{~A}$ & 0.99 & $\mathrm{C} 5 \mathrm{~B}-\mathrm{H} 52 \mathrm{~B}$ & 0.99 \\
\hline $\mathrm{C} 2 \mathrm{~A}-\mathrm{S} 1 \mathrm{~A}-\mathrm{C} 5 \mathrm{~A}$ & $90.24(18)$ & $\mathrm{C} 2 \mathrm{~B}-\mathrm{S} 1 \mathrm{~B}-\mathrm{C} 5 \mathrm{~B}$ & $89.24(18)$ \\
\hline $\mathrm{N} 3 \mathrm{~A}-\mathrm{C} 2 \mathrm{~A}-\mathrm{N} 21 \mathrm{~A}$ & $124.8(3)$ & $\mathrm{N} 3 \mathrm{~B}-\mathrm{C} 2 \mathrm{~B}-\mathrm{N} 21 \mathrm{~B}$ & $125.8(3)$ \\
\hline $\mathrm{N} 3 \mathrm{~A}-\mathrm{C} 2 \mathrm{~A}-\mathrm{S} 1 \mathrm{~A}$ & $117.7(3)$ & $\mathrm{N} 3 \mathrm{~B}-\mathrm{C} 2 \mathrm{~B}-\mathrm{S} 1 \mathrm{~B}$ & $117.1(3)$ \\
\hline $\mathrm{N} 21 \mathrm{~A}-\mathrm{C} 2 \mathrm{~A}-\mathrm{S} 1 \mathrm{~A}$ & $117.5(3)$ & $\mathrm{N} 21 \mathrm{~B}-\mathrm{C} 2 \mathrm{~B}-\mathrm{S} 1 \mathrm{~B}$ & $117.2(3)$ \\
\hline $\mathrm{C} 2 \mathrm{~A}-\mathrm{N} 21 \mathrm{~A}-\mathrm{H} 21 \mathrm{~A}$ & 120.0 & $\mathrm{C} 2 \mathrm{~B}-\mathrm{N} 21 \mathrm{~B}-\mathrm{H} 21 \mathrm{~B}$ & 120.0 \\
\hline $\mathrm{C} 2 \mathrm{~A}-\mathrm{N} 21 \mathrm{~A}-\mathrm{H} 22 \mathrm{~A}$ & 120.0 & $\mathrm{C} 2 \mathrm{~B}-\mathrm{N} 21 \mathrm{~B}-\mathrm{H} 22 \mathrm{~B}$ & 120.0 \\
\hline $\mathrm{H} 21 \mathrm{~A}-\mathrm{N} 21 \mathrm{~A}-\mathrm{H} 22 \mathrm{~A}$ & 120.0 & $\mathrm{H} 21 \mathrm{~B}-\mathrm{N} 21 \mathrm{~B}-\mathrm{H} 22 \mathrm{~B}$ & 120.0 \\
\hline $\mathrm{C} 2 \mathrm{~A}-\mathrm{N} 3 \mathrm{~A}-\mathrm{C} 4 \mathrm{~A}$ & $112.9(3)$ & $\mathrm{C} 2 \mathrm{~B}-\mathrm{N} 3 \mathrm{~B}-\mathrm{C} 4 \mathrm{~B}$ & $112.5(3)$ \\
\hline $\mathrm{N} 3 \mathrm{~A}-\mathrm{C} 4 \mathrm{~A}-\mathrm{C} 5 \mathrm{~A}$ & $112.4(3)$ & $\mathrm{N} 3 \mathrm{~B}-\mathrm{C} 4 \mathrm{~B}-\mathrm{C} 5 \mathrm{~B}$ & $110.9(3)$ \\
\hline $\mathrm{N} 3 \mathrm{~A}-\mathrm{C} 4 \mathrm{~A}-\mathrm{H} 41 \mathrm{~A}$ & 109.1 & $\mathrm{~N} 3 \mathrm{~B}-\mathrm{C} 4 \mathrm{~B}-\mathrm{H} 41 \mathrm{~B}$ & 109.5 \\
\hline $\mathrm{C} 5 \mathrm{~A}-\mathrm{C} 4 \mathrm{~A}-\mathrm{H} 41 \mathrm{~A}$ & 109.1 & $\mathrm{C} 5 \mathrm{~B}-\mathrm{C} 4 \mathrm{~B}-\mathrm{H} 41 \mathrm{~B}$ & 109.5 \\
\hline $\mathrm{N} 3 \mathrm{~A}-\mathrm{C} 4 \mathrm{~A}-\mathrm{H} 42 \mathrm{~A}$ & 109.1 & $\mathrm{~N} 3 \mathrm{~B}-\mathrm{C} 4 \mathrm{~B}-\mathrm{H} 42 \mathrm{~B}$ & 109.5 \\
\hline $\mathrm{C} 5 \mathrm{~A}-\mathrm{C} 4 \mathrm{~A}-\mathrm{H} 42 \mathrm{~A}$ & 109.1 & $\mathrm{C} 5 \mathrm{~B}-\mathrm{C} 4 \mathrm{~B}-\mathrm{H} 42 \mathrm{~B}$ & 109.5 \\
\hline $\mathrm{H} 41 \mathrm{~A}-\mathrm{C} 4 \mathrm{~A}-\mathrm{H} 42 \mathrm{~A}$ & 107.9 & $\mathrm{H} 41 \mathrm{~B}-\mathrm{C} 4 \mathrm{~B}-\mathrm{H} 42 \mathrm{~B}$ & 108.0 \\
\hline $\mathrm{C} 4 \mathrm{~A}-\mathrm{C} 5 \mathrm{~A}-\mathrm{S} 1 \mathrm{~A}$ & $106.7(3)$ & $\mathrm{C} 4 \mathrm{~B}-\mathrm{C} 5 \mathrm{~B}-\mathrm{S} 1 \mathrm{~B}$ & $105.3(3)$ \\
\hline $\mathrm{C} 4 \mathrm{~A}-\mathrm{C} 5 \mathrm{~A}-\mathrm{H} 51 \mathrm{~A}$ & 110.4 & $\mathrm{C} 4 \mathrm{~B}-\mathrm{C} 5 \mathrm{~B}-\mathrm{H} 51 \mathrm{~B}$ & 110.7 \\
\hline $\mathrm{S} 1 \mathrm{~A}-\mathrm{C} 5 \mathrm{~A}-\mathrm{H} 51 \mathrm{~A}$ & 110.4 & $\mathrm{~S} 1 \mathrm{~B}-\mathrm{C} 5 \mathrm{~B}-\mathrm{H} 51 \mathrm{~B}$ & 110.7 \\
\hline $\mathrm{C} 4 \mathrm{~A}-\mathrm{C} 5 \mathrm{~A}-\mathrm{H} 52 \mathrm{~A}$ & 110.4 & $\mathrm{C} 4 \mathrm{~B}-\mathrm{C} 5 \mathrm{~B}-\mathrm{H} 52 \mathrm{~B}$ & 110.7 \\
\hline $\mathrm{S} 1 \mathrm{~A}-\mathrm{C} 5 \mathrm{~A}-\mathrm{H} 52 \mathrm{~A}$ & 110.4 & $\mathrm{~S} 1 \mathrm{~B}-\mathrm{C} 5 \mathrm{~B}-\mathrm{H} 52 \mathrm{~B}$ & 110.7 \\
\hline $\mathrm{H} 51 \mathrm{~A}-\mathrm{C} 5 \mathrm{~A}-\mathrm{H} 52 \mathrm{~A}$ & 108.6 & $\mathrm{H} 51 \mathrm{~B}-\mathrm{C} 5 \mathrm{~B}-\mathrm{H} 52 \mathrm{~B}$ & 108.8 \\
\hline $\mathrm{C} 5 \mathrm{~A}-\mathrm{S} 1 \mathrm{~A}-\mathrm{C} 2 \mathrm{~A}-\mathrm{N} 3 \mathrm{~A}$ & $-2.7(3)$ & $\mathrm{C} 5 \mathrm{~B}-\mathrm{S} 1 \mathrm{~B}-\mathrm{C} 2 \mathrm{~B}-\mathrm{N} 3 \mathrm{~B}$ & $9.6(3)$ \\
\hline $\mathrm{C} 5 \mathrm{~A}-\mathrm{S} 1 \mathrm{~A}-\mathrm{C} 2 \mathrm{~A}-\mathrm{N} 21 \mathrm{~A}$ & $179.3(3)$ & $\mathrm{C} 5 \mathrm{~B}-\mathrm{S} 1 \mathrm{~B}-\mathrm{C} 2 \mathrm{~B}-\mathrm{N} 21 \mathrm{~B}$ & $-170.0(3)$ \\
\hline $\mathrm{N} 21 \mathrm{~A}-\mathrm{C} 2 \mathrm{~A}-\mathrm{N} 3 \mathrm{~A}-\mathrm{C} 4 \mathrm{~A}$ & $179.2(4)$ & $\mathrm{N} 21 \mathrm{~B}-\mathrm{C} 2 \mathrm{~B}-\mathrm{N} 3 \mathrm{~B}-\mathrm{C} 4 \mathrm{~B}$ & $-177.2(4)$ \\
\hline $\mathrm{S} 1 \mathrm{~A}-\mathrm{C} 2 \mathrm{~A}-\mathrm{N} 3 \mathrm{~A}-\mathrm{C} 4 \mathrm{~A}$ & $1.4(4)$ & $\mathrm{S} 1 \mathrm{~B}-\mathrm{C} 2 \mathrm{~B}-\mathrm{N} 3 \mathrm{~B}-\mathrm{C} 4 \mathrm{~B}$ & $3.3(4)$ \\
\hline $\mathrm{C} 2 \mathrm{~A}-\mathrm{N} 3 \mathrm{~A}-\mathrm{C} 4 \mathrm{~A}-\mathrm{C} 5 \mathrm{~A}$ & $1.0(5)$ & $\mathrm{C} 2 \mathrm{~B}-\mathrm{N} 3 \mathrm{~B}-\mathrm{C} 4 \mathrm{~B}-\mathrm{C} 5 \mathrm{~B}$ & $-17.9(5)$ \\
\hline $\mathrm{N} 3 \mathrm{~A}-\mathrm{C} 4 \mathrm{~A}-\mathrm{C} 5 \mathrm{~A}-\mathrm{S} 1 \mathrm{~A}$ & $-2.8(4)$ & $\mathrm{N} 3 \mathrm{~B}-\mathrm{C} 4 \mathrm{~B}-\mathrm{C} 5 \mathrm{~B}-\mathrm{S} 1 \mathrm{~B}$ & $23.4(4)$ \\
\hline $\mathrm{C} 2 \mathrm{~A}-\mathrm{S} 1 \mathrm{~A}-\mathrm{C} 5 \mathrm{~A}-\mathrm{C} 4 \mathrm{~A}$ & $2.9(3)$ & $\mathrm{C} 2 \mathrm{~B}-\mathrm{S} 1 \mathrm{~B}-\mathrm{C} 5 \mathrm{~B}-\mathrm{C} 4 \mathrm{~B}$ & $-17.7(3)$ \\
\hline
\end{tabular}

Hydrogen-bond geometry $\left(\AA,{ }^{\circ}\right)$

\begin{tabular}{lllll}
\hline$D-\mathrm{H} \cdots A$ & $D-\mathrm{H}$ & $\mathrm{H} \cdots A$ & $D \cdots A$ & $D-\mathrm{H} \cdots A$ \\
\hline $\mathrm{N} 21 A-\mathrm{H} 21 A \cdots \mathrm{N} 3 B$ & 0.88 & 2.09 & $2.950(5)$ & 164
\end{tabular}




\begin{tabular}{lllll}
$\mathrm{N} 21 A-\mathrm{H} 22 A \cdots \mathrm{S} 1 B^{\mathrm{i}}$ & 0.88 & 2.75 & $3.575(3)$ & 156 \\
$\mathrm{~N} 21 B-\mathrm{H} 21 B \cdots \mathrm{N} 3 A$ & 0.88 & 2.04 & $2.916(5)$ & 171 \\
$\mathrm{~N} 21 B-\mathrm{H} 22 B \cdots \mathrm{S} 1 A^{\mathrm{ii}}$ & 0.88 & 2.70 & $3.526(3)$ & 156 \\
\hline
\end{tabular}

Symmetry codes: (i) $x-1 / 2,-y+1 / 2, z+1 / 2$; (ii) $x+1 / 2,-y+1 / 2, z-1 / 2$.

\section{(II) 2-Amino-2-thiazolium 2-naphthoxyacetate}

\section{Crystal data}

$\mathrm{C}_{3} \mathrm{H}_{7} \mathrm{~N}_{2} \mathrm{~S}^{+} \cdot \mathrm{C}_{12} \mathrm{H}_{9} \mathrm{O}_{3}{ }^{-}$

$M_{r}=304.36$

Monoclinic, $P 2{ }_{1} / c$

Hall symbol: -P $2 \mathrm{ybc}$

$a=8.3669$ (2) $\AA$

$b=6.3707$ (1) $\AA$

$c=26.3457(6) \AA$

$\beta=92.1992(9)^{\circ}$

$V=1403.27(5) \AA^{3}$

$Z=4$

\section{Data collection}

Nonius KappaCCD area-detector diffractometer

Radiation source: Nonius FR591 rotating anode

Graphite monochromator

Detector resolution: 9.091 pixels $\mathrm{mm}^{-1}$

$\varphi$ and $\omega$ scans

Absorption correction: multi-scan

(SORTAV; Blessing, 1995)

$T_{\text {min }}=0.710, T_{\max }=0.990$

\section{Refinement}

Refinement on $F^{2}$

Least-squares matrix: full

$R\left[F^{2}>2 \sigma\left(F^{2}\right)\right]=0.046$

$w R\left(F^{2}\right)=0.121$

$S=1.02$

3183 reflections

194 parameters

0 restraints

Primary atom site location: structure-invariant direct methods
$F(000)=640$

$D_{\mathrm{x}}=1.441 \mathrm{Mg} \mathrm{m}^{-3}$

Mo $K \alpha$ radiation, $\lambda=0.71073 \AA$

Cell parameters from 4067 reflections

$\theta=2.9-27.5^{\circ}$

$\mu=0.24 \mathrm{~mm}^{-1}$

$T=120 \mathrm{~K}$

Plate, colourless

$0.32 \times 0.10 \times 0.04 \mathrm{~mm}$

15534 measured reflections

3183 independent reflections

2546 reflections with $I>2 \sigma(I)$

$R_{\text {int }}=0.091$

$\theta_{\text {max }}=27.4^{\circ}, \theta_{\min }=3.1^{\circ}$

$h=-10 \rightarrow 10$

$k=-7 \rightarrow 8$

$l=-34 \rightarrow 34$

Secondary atom site location: difference Fourier map

Hydrogen site location: inferred from neighbouring sites

$\mathrm{H}$ atoms treated by a mixture of independent and constrained refinement

$w=1 /\left[\sigma^{2}\left(F_{\mathrm{o}}^{2}\right)+(0.0503 P)^{2}+0.8055 P\right]$ where $P=\left(F_{\mathrm{o}}^{2}+2 F_{\mathrm{c}}^{2}\right) / 3$

$(\Delta / \sigma)_{\max }<0.001$

$\Delta \rho_{\max }=0.25 \mathrm{e} \AA^{-3}$

$\Delta \rho_{\min }=-0.34$ e $\AA^{-3}$

Fractional atomic coordinates and isotropic or equivalent isotropic displacement parameters $\left(\AA^{2}\right)$

\begin{tabular}{lllll}
\hline & $x$ & $y$ & $z$ & $U_{\text {iso }} * / U_{\text {eq }}$ \\
\hline S1A & $-0.19892(6)$ & $-0.56175(8)$ & $0.06199(19)$ & $0.02416(16)$ \\
C2A & $-0.0340(2)$ & $-0.3943(3)$ & $0.06063(7)$ & $0.0173(4)$ \\
N21A & $0.11091(18)$ & $-0.4589(3)$ & $0.07144(6)$ & $0.0198(4)$ \\
H21A & 0.1913 & -0.3698 & 0.0712 & $0.025^{*}$ \\
H22A & 0.1285 & -0.5917 & 0.0790 & $0.025^{*}$ \\
N3A & $-0.07266(19)$ & $-0.1983(3)$ & $0.04883(6)$ & $0.0188(3)$ \\
H3A & $-0.005(3)$ & $-0.097(4)$ & $0.0543(8)$ & $0.023(6)^{*}$
\end{tabular}




$\begin{array}{lllll}\text { C4A } & -0.2446(2) & -0.1539(3) & 0.04747(7) & 0.0218(4) \\ \text { H41A } & -0.2715 & -0.0441 & 0.0220 & 0.027^{*} \\ \text { H42A } & -0.2781 & -0.1050 & 0.0811 & 0.027^{*} \\ \text { C5A } & -0.3271(2) & -0.3597(3) & 0.03314(8) & 0.0228(4) \\ \text { H51A } & -0.3356 & -0.3768 & -0.0042 & 0.029^{*} \\ \text { H52A } & -0.4356 & -0.3655 & 0.0468 & 0.029^{*} \\ \text { C1B } & 0.2758(2) & 0.3025(3) & 0.19187(7) & 0.0194(4) \\ \text { H1B } & 0.2442 & 0.1666 & 0.1805 & 0.024^{*} \\ \text { C2B } & 0.3684(2) & 0.4257(3) & 0.16244(7) & 0.0177(4) \\ \text { C3B } & 0.4200(2) & 0.6263(3) & 0.17909(7) & 0.0206(4) \\ \text { H3B } & 0.4866 & 0.7082 & 0.1584 & 0.026^{*} \\ \text { C4B } & 0.3745(2) & 0.7029(3) & 0.22471(7) & 0.0215(4) \\ \text { H4B } & 0.4095 & 0.8381 & 0.2355 & 0.027^{*} \\ \text { C5B } & 0.2202(2) & 0.6600(3) & 0.30307(7) & 0.0241(4) \\ \text { H5B } & 0.2536 & 0.7948 & 0.3147 & 0.030^{*} \\ \text { C6B } & 0.1201(2) & 0.5432(3) & 0.33145(7) & 0.0258(5) \\ \text { H6B } & 0.0818 & 0.5985 & 0.3622 & 0.032^{*} \\ \text { C7B } & 0.0730(2) & 0.3398(3) & 0.31514(7) & 0.0274(5) \\ \text { H7B } & 0.0042 & 0.2588 & 0.3353 & 0.034^{*} \\ \text { C8B } & 0.1256(2) & 0.2589(3) & 0.27081(7) & 0.0234(4) \\ \text { H8B } & 0.0945 & 0.1213 & 0.2607 & 0.029^{*} \\ \text { C9B } & 0.2266(2) & 0.3787(3) & 0.23966(7) & 0.0201(4) \\ \text { C10B } & 0.2751(2) & 0.5823(3) & 0.25623(7) & 0.0199(4) \\ \text { O11B } & 0.41670(15) & 0.3708(2) & 0.11458(5) & 0.0190(3) \\ \text { C12B } & 0.4426(2) & 0.1542(3) & 0.10439(8) & 0.0209(4) \\ \text { H12B } & 0.4765 & 0.0852 & 0.1367 & 0.026^{*} \\ \text { H13B } & 0.5329 & 0.1433 & 0.0813 & 0.026^{*} \\ \text { C13B } & 0.3018(2) & 0.0295(3) & 0.08086(7) & 0.0177(4) \\ \text { O14B } & 0.16423(15) & 0.1099(2) & 0.07750(5) & 0.0199(3) \\ \text { O15B } & 0.34007(16) & -0.15009(2) & 0.06632(5) & 0.0239(3)\end{array}$

Atomic displacement parameters $\left(\AA^{2}\right)$

\begin{tabular}{lllllll}
\hline & $U^{11}$ & $U^{22}$ & $U^{33}$ & $U^{12}$ & $U^{13}$ & $U^{23}$ \\
\hline S1A & $0.0187(3)$ & $0.0224(3)$ & $0.0313(3)$ & $0.00326(18)$ & $-0.00004(19)$ & $-0.0037(2)$ \\
C2A & $0.0178(9)$ & $0.0186(10)$ & $0.0157(8)$ & $0.0009(7)$ & $0.0024(7)$ & $0.0017(7)$ \\
N21A & $0.0162(8)$ & $0.0173(9)$ & $0.0259(8)$ & $-0.0007(6)$ & $-0.0005(6)$ & $-0.0011(6)$ \\
N3A & $0.0152(8)$ & $0.0182(9)$ & $0.0229(8)$ & $-0.0009(7)$ & $-0.0005(6)$ & $0.0009(7)$ \\
C4A & $0.0170(9)$ & $0.0251(11)$ & $0.0231(10)$ & $-0.0058(8)$ & $-0.0003(7)$ & $0.0008(8)$ \\
C5A & $0.0165(9)$ & $0.0267(11)$ & $0.0253(10)$ & $-0.0024(8)$ & $0.0003(7)$ & $0.0004(8)$ \\
C1B & $0.0177(9)$ & $0.0178(10)$ & $0.0225(10)$ & $0.0003(7)$ & $-0.0019(7)$ & $-0.0003(7)$ \\
C2B & $0.0149(9)$ & $0.0197(10)$ & $0.0185(9)$ & $-0.0031(7)$ & $-0.0014(7)$ & $0.0001(7)$ \\
C3B & $0.0183(9)$ & $0.0208(10)$ & $0.0226(10)$ & $0.0012(7)$ & $-0.0001(7)$ & $-0.0036(8)$ \\
C4B & $0.0213(9)$ & $0.0187(10)$ & $0.0241(10)$ & $0.0010(8)$ & $-0.0026(7)$ & $0.0015(8)$ \\
C5B & $0.0213(10)$ & $0.0300(12)$ & $0.0207(10)$ & $-0.0030(8)$ & $-0.0047(8)$ & $0.0022(8)$ \\
C6B & $0.0224(10)$ & $0.0369(13)$ & $0.0179(10)$ & $-0.0050(8)$ & $-0.0019(8)$ & $0.0009(8)$ \\
C7B & $0.0214(10)$ & $0.0394(13)$ & $0.0213(10)$ & $0.0020(9)$ & $-0.0005(8)$ & $-0.0064(9)$ \\
C8B & $0.0218(10)$ & $0.0263(11)$ & $0.0218(10)$ & $0.0042(8)$ & $-0.0034(7)$ & $-0.0044(8)$
\end{tabular}




\begin{tabular}{lllllll} 
C9B & $0.0150(9)$ & $0.0241(10)$ & $0.0208(9)$ & $-0.0002(7)$ & $-0.0040(7)$ & $-0.0028(8)$ \\
C10B & $0.0163(9)$ & $0.0216(10)$ & $0.0214(9)$ & $-0.0031(7)$ & $-0.0039(7)$ & $-0.0008(8)$ \\
O11B & $0.0194(7)$ & $0.0172(7)$ & $0.0206(7)$ & $-0.0004(5)$ & $0.0032(5)$ & $0.0014(5)$ \\
C12B & $0.0163(9)$ & $0.0188(10)$ & $0.0277(10)$ & $-0.0017(7)$ & $0.0019(7)$ & $0.0023(8)$ \\
C13B & $0.0175(9)$ & $0.0190(10)$ & $0.0167(9)$ & $-0.0012(7)$ & $0.0042(7)$ & $-0.0029(7)$ \\
O14B & $0.0152(6)$ & $0.0200(7)$ & $0.0245(7)$ & $-0.0006(5)$ & $0.0001(5)$ & $0.0008(5)$ \\
O15B & $0.0221(7)$ & $0.0189(8)$ & $0.0309(8)$ & $-0.0016(5)$ & $0.0018(6)$ & $0.0038(6)$ \\
\hline
\end{tabular}

Geometric parameters $\left(\AA,{ }^{\circ}\right)$

\begin{tabular}{|c|c|c|c|}
\hline $\mathrm{S} 1 \mathrm{~A}-\mathrm{C} 2 \mathrm{~A}$ & $1.7455(19)$ & $\mathrm{C} 3 \mathrm{~B}-\mathrm{H} 3 \mathrm{~B}$ & 0.95 \\
\hline $\mathrm{S} 1 \mathrm{~A}-\mathrm{C} 5 \mathrm{~A}$ & $1.823(2)$ & $\mathrm{C} 4 \mathrm{~B}-\mathrm{C} 10 \mathrm{~B}$ & $1.423(3)$ \\
\hline $\mathrm{C} 2 \mathrm{~A}-\mathrm{N} 21 \mathrm{~A}$ & $1.302(2)$ & $\mathrm{C} 4 \mathrm{~B}-\mathrm{H} 4 \mathrm{~B}$ & 0.95 \\
\hline $\mathrm{C} 2 \mathrm{~A}-\mathrm{N} 3 \mathrm{~A}$ & $1.324(2)$ & $\mathrm{C} 5 \mathrm{~B}-\mathrm{C} 6 \mathrm{~B}$ & $1.365(3)$ \\
\hline $\mathrm{N} 21 \mathrm{~A}-\mathrm{H} 21 \mathrm{~A}$ & 0.88 & $\mathrm{C} 5 \mathrm{~B}-\mathrm{C} 10 \mathrm{~B}$ & $1.422(3)$ \\
\hline $\mathrm{N} 21 \mathrm{~A}-\mathrm{H} 22 \mathrm{~A}$ & 0.88 & $\mathrm{C} 5 \mathrm{~B}-\mathrm{H} 5 \mathrm{~B}$ & 0.95 \\
\hline $\mathrm{N} 3 \mathrm{~A}-\mathrm{C} 4 \mathrm{~A}$ & $1.465(2)$ & $\mathrm{C} 6 \mathrm{~B}-\mathrm{C} 7 \mathrm{~B}$ & $1.417(3)$ \\
\hline $\mathrm{N} 3 \mathrm{~A}-\mathrm{H} 3 \mathrm{~A}$ & $0.87(2)$ & $\mathrm{C} 6 \mathrm{~B}-\mathrm{H} 6 \mathrm{~B}$ & 0.95 \\
\hline $\mathrm{C} 4 \mathrm{~A}-\mathrm{C} 5 \mathrm{~A}$ & $1.523(3)$ & $\mathrm{C} 7 \mathrm{~B}-\mathrm{C} 8 \mathrm{~B}$ & $1.365(3)$ \\
\hline $\mathrm{C} 4 \mathrm{~A}-\mathrm{H} 41 \mathrm{~A}$ & 0.99 & $\mathrm{C} 7 \mathrm{~B}-\mathrm{H} 7 \mathrm{~B}$ & 0.95 \\
\hline $\mathrm{C} 4 \mathrm{~A}-\mathrm{H} 42 \mathrm{~A}$ & 0.99 & $\mathrm{C} 8 \mathrm{~B}-\mathrm{C} 9 \mathrm{~B}$ & $1.422(3)$ \\
\hline $\mathrm{C} 5 \mathrm{~A}-\mathrm{H} 51 \mathrm{~A}$ & 0.99 & $\mathrm{C} 8 \mathrm{~B}-\mathrm{H} 8 \mathrm{~B}$ & 0.95 \\
\hline $\mathrm{C} 5 \mathrm{~A}-\mathrm{H} 52 \mathrm{~A}$ & 0.99 & $\mathrm{C} 9 \mathrm{~B}-\mathrm{C} 10 \mathrm{~B}$ & $1.423(3)$ \\
\hline $\mathrm{C} 1 \mathrm{~B}-\mathrm{C} 2 \mathrm{~B}$ & $1.365(3)$ & $\mathrm{O} 11 \mathrm{~B}-\mathrm{C} 12 \mathrm{~B}$ & $1.424(2)$ \\
\hline $\mathrm{C} 1 \mathrm{~B}-\mathrm{C} 9 \mathrm{~B}$ & $1.425(3)$ & $\mathrm{C} 12 \mathrm{~B}-\mathrm{C} 13 \mathrm{~B}$ & $1.532(3)$ \\
\hline $\mathrm{C} 1 \mathrm{~B}-\mathrm{H} 1 \mathrm{~B}$ & 0.95 & $\mathrm{C} 12 \mathrm{~B}-\mathrm{H} 12 \mathrm{~B}$ & 0.99 \\
\hline $\mathrm{C} 2 \mathrm{~B}-\mathrm{O} 11 \mathrm{~B}$ & $1.384(2)$ & $\mathrm{C} 12 \mathrm{~B}-\mathrm{H} 13 \mathrm{~B}$ & 0.99 \\
\hline $\mathrm{C} 2 \mathrm{~B}-\mathrm{C} 3 \mathrm{~B}$ & $1.413(3)$ & $\mathrm{C} 13 \mathrm{~B}-\mathrm{O} 15 \mathrm{~B}$ & $1.252(2)$ \\
\hline $\mathrm{C} 3 \mathrm{~B}-\mathrm{C} 4 \mathrm{~B}$ & $1.365(3)$ & $\mathrm{C} 13 \mathrm{~B}-\mathrm{O} 14 \mathrm{~B}$ & $1.260(2)$ \\
\hline $\mathrm{C} 2 \mathrm{~A}-\mathrm{S} 1 \mathrm{~A}-\mathrm{C} 5 \mathrm{~A}$ & $90.73(9)$ & $\mathrm{C} 3 \mathrm{~B}-\mathrm{C} 4 \mathrm{~B}-\mathrm{C} 10 \mathrm{~B}$ & $120.65(18)$ \\
\hline $\mathrm{N} 21 \mathrm{~A}-\mathrm{C} 2 \mathrm{~A}-\mathrm{N} 3 \mathrm{~A}$ & $124.53(17)$ & $\mathrm{C} 3 \mathrm{~B}-\mathrm{C} 4 \mathrm{~B}-\mathrm{H} 4 \mathrm{~B}$ & 119.7 \\
\hline $\mathrm{N} 21 \mathrm{~A}-\mathrm{C} 2 \mathrm{~A}-\mathrm{S} 1 \mathrm{~A}$ & $122.19(15)$ & $\mathrm{C} 10 \mathrm{~B}-\mathrm{C} 4 \mathrm{~B}-\mathrm{H} 4 \mathrm{~B}$ & 119.7 \\
\hline $\mathrm{N} 3 \mathrm{~A}-\mathrm{C} 2 \mathrm{~A}-\mathrm{S} 1 \mathrm{~A}$ & $113.27(14)$ & $\mathrm{C} 6 \mathrm{~B}-\mathrm{C} 5 \mathrm{~B}-\mathrm{C} 10 \mathrm{~B}$ & $120.7(2)$ \\
\hline $\mathrm{C} 2 \mathrm{~A}-\mathrm{N} 21 \mathrm{~A}-\mathrm{H} 21 \mathrm{~A}$ & 120.0 & $\mathrm{C} 6 \mathrm{~B}-\mathrm{C} 5 \mathrm{~B}-\mathrm{H} 5 \mathrm{~B}$ & 119.6 \\
\hline $\mathrm{C} 2 \mathrm{~A}-\mathrm{N} 21 \mathrm{~A}-\mathrm{H} 22 \mathrm{~A}$ & 120.0 & $\mathrm{C} 10 \mathrm{~B}-\mathrm{C} 5 \mathrm{~B}-\mathrm{H} 5 \mathrm{~B}$ & 119.6 \\
\hline $\mathrm{H} 21 \mathrm{~A}-\mathrm{N} 21 \mathrm{~A}-\mathrm{H} 22 \mathrm{~A}$ & 120.0 & $\mathrm{C} 5 \mathrm{~B}-\mathrm{C} 6 \mathrm{~B}-\mathrm{C} 7 \mathrm{~B}$ & $120.13(19)$ \\
\hline $\mathrm{C} 2 \mathrm{~A}-\mathrm{N} 3 \mathrm{~A}-\mathrm{C} 4 \mathrm{~A}$ & $114.77(16)$ & $\mathrm{C} 5 \mathrm{~B}-\mathrm{C} 6 \mathrm{~B}-\mathrm{H} 6 \mathrm{~B}$ & 119.9 \\
\hline $\mathrm{C} 2 \mathrm{~A}-\mathrm{N} 3 \mathrm{~A}-\mathrm{H} 3 \mathrm{~A}$ & $120.5(15)$ & $\mathrm{C} 7 \mathrm{~B}-\mathrm{C} 6 \mathrm{~B}-\mathrm{H} 6 \mathrm{~B}$ & 119.9 \\
\hline $\mathrm{C} 4 \mathrm{~A}-\mathrm{N} 3 \mathrm{~A}-\mathrm{H} 3 \mathrm{~A}$ & $120.0(15)$ & $\mathrm{C} 8 \mathrm{~B}-\mathrm{C} 7 \mathrm{~B}-\mathrm{C} 6 \mathrm{~B}$ & $120.66(19)$ \\
\hline $\mathrm{N} 3 \mathrm{~A}-\mathrm{C} 4 \mathrm{~A}-\mathrm{C} 5 \mathrm{~A}$ & $105.96(15)$ & $\mathrm{C} 8 \mathrm{~B}-\mathrm{C} 7 \mathrm{~B}-\mathrm{H} 7 \mathrm{~B}$ & 119.7 \\
\hline $\mathrm{N} 3 \mathrm{~A}-\mathrm{C} 4 \mathrm{~A}-\mathrm{H} 41 \mathrm{~A}$ & 110.5 & $\mathrm{C} 6 \mathrm{~B}-\mathrm{C} 7 \mathrm{~B}-\mathrm{H} 7 \mathrm{~B}$ & 119.7 \\
\hline $\mathrm{C} 5 \mathrm{~A}-\mathrm{C} 4 \mathrm{~A}-\mathrm{H} 41 \mathrm{~A}$ & 110.5 & $\mathrm{C} 7 \mathrm{~B}-\mathrm{C} 8 \mathrm{~B}-\mathrm{C} 9 \mathrm{~B}$ & $120.64(19)$ \\
\hline $\mathrm{N} 3 \mathrm{~A}-\mathrm{C} 4 \mathrm{~A}-\mathrm{H} 42 \mathrm{~A}$ & 110.5 & $\mathrm{C} 7 \mathrm{~B}-\mathrm{C} 8 \mathrm{~B}-\mathrm{H} 8 \mathrm{~B}$ & 119.7 \\
\hline $\mathrm{C} 5 \mathrm{~A}-\mathrm{C} 4 \mathrm{~A}-\mathrm{H} 42 \mathrm{~A}$ & 110.5 & $\mathrm{C} 9 \mathrm{~B}-\mathrm{C} 8 \mathrm{~B}-\mathrm{H} 8 \mathrm{~B}$ & 119.7 \\
\hline $\mathrm{H} 41 \mathrm{~A}-\mathrm{C} 4 \mathrm{~A}-\mathrm{H} 42 \mathrm{~A}$ & 108.7 & $\mathrm{C} 8 \mathrm{~B}-\mathrm{C} 9 \mathrm{~B}-\mathrm{C} 10 \mathrm{~B}$ & $118.74(18)$ \\
\hline $\mathrm{C} 4 \mathrm{~A}-\mathrm{C} 5 \mathrm{~A}-\mathrm{S} 1 \mathrm{~A}$ & $104.53(12)$ & $\mathrm{C} 8 \mathrm{~B}-\mathrm{C} 9 \mathrm{~B}-\mathrm{C} 1 \mathrm{~B}$ & $121.69(18)$ \\
\hline $\mathrm{C} 4 \mathrm{~A}-\mathrm{C} 5 \mathrm{~A}-\mathrm{H} 51 \mathrm{~A}$ & 110.8 & $\mathrm{C} 10 \mathrm{~B}-\mathrm{C} 9 \mathrm{~B}-\mathrm{C} 1 \mathrm{~B}$ & $119.54(17)$ \\
\hline
\end{tabular}




\begin{tabular}{|c|c|c|c|}
\hline $\mathrm{S} 1 \mathrm{~A}-\mathrm{C} 5 \mathrm{~A}-\mathrm{H} 51 \mathrm{~A}$ & 110.8 & $\mathrm{C} 5 \mathrm{~B}-\mathrm{C} 10 \mathrm{~B}-\mathrm{C} 9 \mathrm{~B}$ & $119.08(18)$ \\
\hline $\mathrm{C} 4 \mathrm{~A}-\mathrm{C} 5 \mathrm{~A}-\mathrm{H} 52 \mathrm{~A}$ & 110.8 & $\mathrm{C} 5 \mathrm{~B}-\mathrm{C} 10 \mathrm{~B}-\mathrm{C} 4 \mathrm{~B}$ & $122.26(18)$ \\
\hline $\mathrm{S} 1 \mathrm{~A}-\mathrm{C} 5 \mathrm{~A}-\mathrm{H} 52 \mathrm{~A}$ & 110.8 & $\mathrm{C} 9 \mathrm{~B}-\mathrm{C} 10 \mathrm{~B}-\mathrm{C} 4 \mathrm{~B}$ & $118.65(17)$ \\
\hline $\mathrm{H} 51 \mathrm{~A}-\mathrm{C} 5 \mathrm{~A}-\mathrm{H} 52 \mathrm{~A}$ & 108.9 & $\mathrm{C} 2 \mathrm{~B}-\mathrm{O} 11 \mathrm{~B}-\mathrm{C} 12 \mathrm{~B}$ & $117.93(14)$ \\
\hline $\mathrm{C} 2 \mathrm{~B}-\mathrm{C} 1 \mathrm{~B}-\mathrm{C} 9 \mathrm{~B}$ & $119.71(18)$ & $\mathrm{O} 11 \mathrm{~B}-\mathrm{C} 12 \mathrm{~B}-\mathrm{C} 13 \mathrm{~B}$ & $117.32(15)$ \\
\hline $\mathrm{C} 2 \mathrm{~B}-\mathrm{C} 1 \mathrm{~B}-\mathrm{H} 1 \mathrm{~B}$ & 120.1 & $\mathrm{O} 11 \mathrm{~B}-\mathrm{C} 12 \mathrm{~B}-\mathrm{H} 12 \mathrm{~B}$ & 108.0 \\
\hline $\mathrm{C} 9 \mathrm{~B}-\mathrm{C} 1 \mathrm{~B}-\mathrm{H} 1 \mathrm{~B}$ & 120.1 & $\mathrm{C} 13 \mathrm{~B}-\mathrm{C} 12 \mathrm{~B}-\mathrm{H} 12 \mathrm{~B}$ & 108.0 \\
\hline $\mathrm{C} 1 \mathrm{~B}-\mathrm{C} 2 \mathrm{~B}-\mathrm{O} 11 \mathrm{~B}$ & $124.35(17)$ & $\mathrm{O} 11 \mathrm{~B}-\mathrm{C} 12 \mathrm{~B}-\mathrm{H} 13 \mathrm{~B}$ & 108.0 \\
\hline $\mathrm{C} 1 \mathrm{~B}-\mathrm{C} 2 \mathrm{~B}-\mathrm{C} 3 \mathrm{~B}$ & $121.10(17)$ & $\mathrm{C} 13 \mathrm{~B}-\mathrm{C} 12 \mathrm{~B}-\mathrm{H} 13 \mathrm{~B}$ & 108.0 \\
\hline $\mathrm{O} 11 \mathrm{~B}-\mathrm{C} 2 \mathrm{~B}-\mathrm{C} 3 \mathrm{~B}$ & $114.53(16)$ & $\mathrm{H} 12 \mathrm{~B}-\mathrm{C} 12 \mathrm{~B}-\mathrm{H} 13 \mathrm{~B}$ & 107.2 \\
\hline $\mathrm{C} 4 \mathrm{~B}-\mathrm{C} 3 \mathrm{~B}-\mathrm{C} 2 \mathrm{~B}$ & $120.32(18)$ & $\mathrm{O} 15 \mathrm{~B}-\mathrm{C} 13 \mathrm{~B}-\mathrm{O} 14 \mathrm{~B}$ & $126.43(17)$ \\
\hline $\mathrm{C} 4 \mathrm{~B}-\mathrm{C} 3 \mathrm{~B}-\mathrm{H} 3 \mathrm{~B}$ & 119.8 & $\mathrm{O} 15 \mathrm{~B}-\mathrm{C} 13 \mathrm{~B}-\mathrm{C} 12 \mathrm{~B}$ & $113.33(15)$ \\
\hline $\mathrm{C} 2 \mathrm{~B}-\mathrm{C} 3 \mathrm{~B}-\mathrm{H} 3 \mathrm{~B}$ & 119.8 & $\mathrm{O} 14 \mathrm{~B}-\mathrm{C} 13 \mathrm{~B}-\mathrm{C} 12 \mathrm{~B}$ & $120.24(16)$ \\
\hline $\mathrm{C} 5 \mathrm{~A}-\mathrm{S} 1 \mathrm{~A}-\mathrm{C} 2 \mathrm{~A}-\mathrm{N} 21 \mathrm{~A}$ & $-171.09(16)$ & $\mathrm{C} 7 \mathrm{~B}-\mathrm{C} 8 \mathrm{~B}-\mathrm{C} 9 \mathrm{~B}-\mathrm{C} 1 \mathrm{~B}$ & $-176.41(17)$ \\
\hline $\mathrm{C} 5 \mathrm{~A}-\mathrm{S} 1 \mathrm{~A}-\mathrm{C} 2 \mathrm{~A}-\mathrm{N} 3 \mathrm{~A}$ & $10.36(15)$ & $\mathrm{C} 2 \mathrm{~B}-\mathrm{C} 1 \mathrm{~B}-\mathrm{C} 9 \mathrm{~B}-\mathrm{C} 8 \mathrm{~B}$ & $177.84(17)$ \\
\hline $\mathrm{N} 21 \mathrm{~A}-\mathrm{C} 2 \mathrm{~A}-\mathrm{N} 3 \mathrm{~A}-\mathrm{C} 4 \mathrm{~A}$ & $-169.07(17)$ & $\mathrm{C} 2 \mathrm{~B}-\mathrm{C} 1 \mathrm{~B}-\mathrm{C} 9 \mathrm{~B}-\mathrm{C} 10 \mathrm{~B}$ & $-0.2(3)$ \\
\hline $\mathrm{S} 1 \mathrm{~A}-\mathrm{C} 2 \mathrm{~A}-\mathrm{N} 3 \mathrm{~A}-\mathrm{C} 4 \mathrm{~A}$ & $9.4(2)$ & $\mathrm{C} 6 \mathrm{~B}-\mathrm{C} 5 \mathrm{~B}-\mathrm{C} 10 \mathrm{~B}-\mathrm{C} 9 \mathrm{~B}$ & $-1.2(3)$ \\
\hline $\mathrm{C} 2 \mathrm{~A}-\mathrm{N} 3 \mathrm{~A}-\mathrm{C} 4 \mathrm{~A}-\mathrm{C} 5 \mathrm{~A}$ & $-28.8(2)$ & $\mathrm{C} 6 \mathrm{~B}-\mathrm{C} 5 \mathrm{~B}-\mathrm{C} 10 \mathrm{~B}-\mathrm{C} 4 \mathrm{~B}$ & $177.48(17)$ \\
\hline $\mathrm{N} 3 \mathrm{~A}-\mathrm{C} 4 \mathrm{~A}-\mathrm{C} 5 \mathrm{~A}-\mathrm{S} 1 \mathrm{~A}$ & $33.38(17)$ & $\mathrm{C} 8 \mathrm{~B}-\mathrm{C} 9 \mathrm{~B}-\mathrm{C} 10 \mathrm{~B}-\mathrm{C} 5 \mathrm{~B}$ & $-0.5(3)$ \\
\hline $\mathrm{C} 2 \mathrm{~A}-\mathrm{S} 1 \mathrm{~A}-\mathrm{C} 5 \mathrm{~A}-\mathrm{C} 4 \mathrm{~A}$ & $-25.22(14)$ & $\mathrm{C} 1 \mathrm{~B}-\mathrm{C} 9 \mathrm{~B}-\mathrm{C} 10 \mathrm{~B}-\mathrm{C} 5 \mathrm{~B}$ & $177.55(16)$ \\
\hline $\mathrm{C} 9 \mathrm{~B}-\mathrm{C} 1 \mathrm{~B}-\mathrm{C} 2 \mathrm{~B}-\mathrm{O} 11 \mathrm{~B}$ & $-177.00(16)$ & $\mathrm{C} 8 \mathrm{~B}-\mathrm{C} 9 \mathrm{~B}-\mathrm{C} 10 \mathrm{~B}-\mathrm{C} 4 \mathrm{~B}$ & $-179.21(17)$ \\
\hline $\mathrm{C} 9 \mathrm{~B}-\mathrm{C} 1 \mathrm{~B}-\mathrm{C} 2 \mathrm{~B}-\mathrm{C} 3 \mathrm{~B}$ & $1.5(3)$ & $\mathrm{C} 1 \mathrm{~B}-\mathrm{C} 9 \mathrm{~B}-\mathrm{C} 10 \mathrm{~B}-\mathrm{C} 4 \mathrm{~B}$ & $-1.1(3)$ \\
\hline $\mathrm{C} 1 \mathrm{~B}-\mathrm{C} 2 \mathrm{~B}-\mathrm{C} 3 \mathrm{~B}-\mathrm{C} 4 \mathrm{~B}$ & $-1.5(3)$ & $\mathrm{C} 3 \mathrm{~B}-\mathrm{C} 4 \mathrm{~B}-\mathrm{C} 10 \mathrm{~B}-\mathrm{C} 5 \mathrm{~B}$ & $-177.51(17)$ \\
\hline $\mathrm{O} 11 \mathrm{~B}-\mathrm{C} 2 \mathrm{~B}-\mathrm{C} 3 \mathrm{~B}-\mathrm{C} 4 \mathrm{~B}$ & $177.12(16)$ & $\mathrm{C} 3 \mathrm{~B}-\mathrm{C} 4 \mathrm{~B}-\mathrm{C} 10 \mathrm{~B}-\mathrm{C} 9 \mathrm{~B}$ & $1.1(3)$ \\
\hline $\mathrm{C} 2 \mathrm{~B}-\mathrm{C} 3 \mathrm{~B}-\mathrm{C} 4 \mathrm{~B}-\mathrm{C} 10 \mathrm{~B}$ & $0.2(3)$ & $\mathrm{C} 1 \mathrm{~B}-\mathrm{C} 2 \mathrm{~B}-\mathrm{O} 11 \mathrm{~B}-\mathrm{C} 12 \mathrm{~B}$ & $-31.7(2)$ \\
\hline $\mathrm{C} 10 \mathrm{~B}-\mathrm{C} 5 \mathrm{~B}-\mathrm{C} 6 \mathrm{~B}-\mathrm{C} 7 \mathrm{~B}$ & $1.8(3)$ & $\mathrm{C} 3 \mathrm{~B}-\mathrm{C} 2 \mathrm{~B}-\mathrm{O} 11 \mathrm{~B}-\mathrm{C} 12 \mathrm{~B}$ & $149.67(16)$ \\
\hline $\mathrm{C} 5 \mathrm{~B}-\mathrm{C} 6 \mathrm{~B}-\mathrm{C} 7 \mathrm{~B}-\mathrm{C} 8 \mathrm{~B}$ & $-0.6(3)$ & $\mathrm{C} 2 \mathrm{~B}-\mathrm{O} 11 \mathrm{~B}-\mathrm{C} 12 \mathrm{~B}-\mathrm{C} 13 \mathrm{~B}$ & $92.8(2)$ \\
\hline $\mathrm{C} 6 \mathrm{~B}-\mathrm{C} 7 \mathrm{~B}-\mathrm{C} 8 \mathrm{~B}-\mathrm{C} 9 \mathrm{~B}$ & $-1.1(3)$ & $\mathrm{O} 11 \mathrm{~B}-\mathrm{C} 12 \mathrm{~B}-\mathrm{C} 13 \mathrm{~B}-\mathrm{O} 15 \mathrm{~B}$ & $170.22(16)$ \\
\hline $\mathrm{C} 7 \mathrm{~B}-\mathrm{C} 8 \mathrm{~B}-\mathrm{C} 9 \mathrm{~B}-\mathrm{C} 10 \mathrm{~B}$ & $1.6(3)$ & $\mathrm{O} 11 \mathrm{~B}-\mathrm{C} 12 \mathrm{~B}-\mathrm{C} 13 \mathrm{~B}-\mathrm{O} 14 \mathrm{~B}$ & $-9.5(3)$ \\
\hline
\end{tabular}

Hydrogen-bond geometry $\left(A,{ }^{\circ}\right)$

\begin{tabular}{lllll}
\hline$D-\mathrm{H} \cdots A$ & $D-\mathrm{H}$ & $\mathrm{H} \cdots A$ & $D \cdots A$ & $D-\mathrm{H} \cdots A$ \\
\hline $\mathrm{N} 21 A-\mathrm{H} 21 A \cdots \mathrm{O} 15 B$ & 0.88 & 1.88 & $2.754(2)$ & 171 \\
$\mathrm{~N} 21 A-\mathrm{H} 22 A \cdots \mathrm{O} 14 B^{\mathrm{i}}$ & 0.88 & 1.93 & $2.787(2)$ & 166 \\
$\mathrm{~N} 21 A-\mathrm{H} 22 A \cdots \mathrm{O} 11 B^{\mathrm{i}}$ & 0.88 & 2.56 & $2.965(2)$ & 109 \\
$\mathrm{~N} 3 A-\mathrm{H} 3 A \cdots \mathrm{O} 14 B$ & $0.87(2)$ & $2.01(2)$ & $2.871(2)$ & $170(2)$ \\
\hline
\end{tabular}

Symmetry code: (i) $x, y-1, z$. 